\title{
Design of a Low-Energy FARAD Thruster
}

\author{
K.A. Polzin* \\ NASA-Marshall Space Flight Center, Huntsville, AL 35812 \\ M.F. Rose ${ }^{\dagger}$ and R. Miller \\ S. Best $\$$ \\ Radiance Technologies, Huntsville, AL 35805 \\ Auburn University, Auburn, AL 36849

\section{T. Owens $\uparrow$} \\ West Virginia High Technology Consortium Foundation, Fairmont, WV 26554 \\ J. Dankanich"l \\ Gray Research, Inc., Huntsville, AL 35806
}

The design of an electrodeless thruster that relies on a pulsed, rf-assisted discharge and electromagnetic acceleration using an inductive coil is presented. The thruster design is optimized using known performance, scaling parameters and experimentally-determined design rules, with design targets for discharge energy, plasma exhaust velocity, and thrust efficiency of $100 \mathrm{~J} /$ pulse, $25 \mathrm{~km} / \mathrm{s}$, and $50 \%$, respectively. Propellant is injected using a high-speed gas valve and preionized by a pulsed-RF signal supplied by a vector inversion generator, allowing for current sheet formation at lower discharge voltages and energies relative to pulsed inductive accelerators that do not employ preionization. The acceleration coil is designed to possess an inductance of at least $700 \mathrm{nH}$ while the target stray (noncoil) inductance in the circuit is $70 \mathrm{nH}$. A Bernardes and Merryman pulsed power train or a pulse compression power train provide current to the acceleration coil and solid-state components are used to switch both powertrains.

\section{Nomenclature}

$A_{c} \quad$ magnetic core area, $\mathrm{m}^{2}$

$B_{s} \quad$ saturation magnetic field, $\mathrm{T}$

$C$ capacitance, $\mathrm{F}$

I current, A

ID inner diameter, $m$

$j \quad$ linear current density, $\mathrm{A} / \mathrm{m}$

$\mathcal{L}$. coil width, m

$\dot{L} \quad$ dynamic impedance, $\mathrm{H} / \mathrm{s}$

$L^{\prime} \quad$ inductance per unit length, $\mathrm{H} / \mathrm{m}$

$L^{*} \quad$ inductance ratio

$L_{0} \quad$ stray inductance, $\mathrm{H}$

$L_{C} \quad$ coil inductance, $\mathrm{H}$.

$\begin{array}{ll}m_{\text {bit }} & \text { mass bit, } \mathrm{kg} / \mathrm{pulse} \\ n & \text { number of turns } \\ \mathrm{OD} & \text { outer diameter, } \mathrm{m} \\ t & \text { time, } \mathrm{s} \\ V & \text { voltage, } \mathrm{V} \\ V_{0} & \text { initial charge voltage, } \mathrm{V} \\ v_{z} & \text { exhaust velocity, } \mathrm{m} / \mathrm{s} \\ z & \text { axial distance, } \mathrm{m} \\ z_{0} & \text { electromagnetic decoupling distance, } \mathrm{m} \\ \alpha & \text { dynamic impedance parameter } \\ \eta_{t} & \text { thrust efficiency }\end{array}$

\footnotetext{
*Propulsion Research Engineer, Nuclear Systems Branch, Propulsion Systems Department. Member AIAA.

†V.P. of Research, Auburn Office, 231 Leach Science Center, Auburn University, Auburn, AL 36849. Associate Fellow AIAA.

$\ddagger$ †roject Manager, Auburn Office, 231 Leach Science Center, Auburn University, Auburn, AL 36849. Member AIAA.

${ }^{\S}$ Researcher, Space Research Institute, 231 Leach Science Center. Member AIAA.

TPrincipal Scientist, WVHTCF Scientific Research Group.

"Lead Systems Engineer, In-Space Propulsion. Member AIAA.

This material is declared a work of the U.S. Government and is not subject to copyright protection in the United States.
} 


\section{Introduction}

PULSED inductive plasma accelerators are spacecraft propulsion devices in which energy is stored in a P capacitor and then discharged through an inductive coil. The device is electrodeless, inducing a current in a plasma located near the face of the coil. The propellant is accelerated and expelled at a high exhaust velocity $(\mathcal{O}(10 \mathrm{~km} / \mathrm{s}))$ by the Lorentz body force arising from the interaction of the plasma current and the induced magnetic field. Presently, there are two concepts which operate on this principle. One is the Pulsed Inductive Thruster (PIT), ${ }^{1}$ in which both propellant ionization and acceleration are performed by the pulse of current flowing through the inductive coil. In contrast, the Faraday Accelerator with Radio-frequency Assisted Discharge (FARAD) $)^{2}$ uses a separate inductive discharge to preionize the propellant before it is accelerated by the current pulse in the coil.

Inductive plasma accelerators are attractive as propulsive devices for many reasons. The lifetime and contamination issues associated with electrode erosion in conventional pulsed plasma thrusters (PPTs) do not exist in devices where the discharge is inductively driven. In addition, a wider variety of propellants (e.g. $\mathrm{CO}_{2}, \mathrm{H}_{2} \mathrm{O}$ ) becomes available for use when compatibility with metallic electrodes is no longer an issue. Moreover, pulsed inductive accelerators (indeed, pulsed accelerators in general) can maintain the same performance level over a wide range of input power levels by adjusting the pulse rate.

The state-of-the-art PIT thruster is $1 \mathrm{~m}$ in diameter and requires approximately $4 \mathrm{~kJ} /$ pulse for efficient operation (using ammonia as a propellant). ${ }^{1}$ In addition, this thruster has multiple capacitors which must be individually (and simultaneously) discharged to produce the azimuthal current in the coil. Recently, a FARAD-based benchtop accelerator was used to demonstrate that preionization of the propellant using a steady-state helicon discharge allows for formation and acceleration of an inductive current sheet at much lower discharge energies and voltages than those found in the PIT. ${ }^{2,3}$ While the experimental apparatus in that study was not optimized as a thruster, the work resulted in the analytical derivation of performance scaling parameters and the formulation of a set of design rules for a FARAD thruster. ${ }^{3-5}$.

In this paper, we present the design of a complete laboratory-model FARAD thruster with all the subsystems (mass injection, preionization, and acceleration) integrated into a single unit. The system uses a pulsed, ringing $R F$-frequency signal produced by a discharging vector inversion generator (VIG) to preionize the gas. The acceleration stage operates on the order of $100 \mathrm{~J} /$ pulse and can be driven by several different pulsed powertrains. These include a simple capacitor coupled to the system, a Bernardes and Merryman based drive circuit, and a pulse-compression circuit that takes a temporally broad, low-current pulse and transforms it into a short, high-current pulse.

The outline for the rest of this paper is as follows. In Sect. II we review the scaling parameters and design rules for a FARAD thruster and show how we arrived at our design. The designs of the individual subsystems in our FARAD thruster are discussed in Sect. III. Any data obtained or modeling performed to test certain subsystems and validate our design approach are included in this section as well.

\section{Scaling and Design Considerations}

The inductive coil and its associated drive circuit are optimized for plasma acceleration and current sheet formation. From the very beginning, we assumed a discharge energy of $100 \mathrm{~J} /$ pulse and fixed our acceleration coil dimensions with an inner diameter of $12 \mathrm{~cm}$ and an outer diameter of $30 \mathrm{~cm}$. We proceed with a discussion of the performance scaling parameters and the optimization procedure.

\section{A. Acceleration Optimization}

Optimization of the acceleration process follows directly from a set of recently derived performance scaling parameters. ${ }^{4}$ Specifically, we focus on the dynamic impedance parameter, $\alpha$, which is defined in terms of measurable quantities as

$$
\alpha=\frac{C^{2} V_{0}^{2} L_{C}}{2 m_{\mathrm{bit}} z_{0}^{2}}
$$

where $C$ is the capacitance in the bank, $V_{0}$ is the charge voltage, $L_{C}$ is the inductance of the coil, $m_{\mathrm{bit}}$ is the mass bit or propellant mass expended in a single pulse, and $z_{0}$ is the decoupling distance or length scale 
over which inductive plasma acceleration occurs. The parameter $\alpha$ can be recast as

$$
\alpha=\frac{1}{8 \pi^{2}} \frac{C V_{0}^{2} / 2}{m_{\mathrm{bit}} v_{z}^{2} / 2} L^{*}\left(\frac{2 \pi \sqrt{L_{0} C}}{L_{0} / \dot{L}}\right)^{2},
$$

where $v_{z}$ is the propellant exhaust velocity, $L_{0}$ is the stray (non-coil) inductance in the circuit, $L^{*}$ is the inductance ratio $L_{0} / L_{C}$, and $\dot{L}$ is the dynamic impedance which is defined as $v_{z} L^{\prime}$ where $L^{\prime}$ is an effective inductance per unit length equal to $L_{C} / z_{0}$. We recognize several important ratios in Eq. (2), specifically the inverse of thrust efficiency (defined as the ratio of directed jet kinetic energy to input electrical energy); the Lovberg ratio, ${ }^{6}$ which is $1 / L^{*}$; and the ratio of the resonant period of the unloaded circuit (i.e. without the coil inductance) to the time scale that the current sheet remains in the acceleration region before decoupling from the coil. The last ratio can also be understood as a quantitative measure of the transfer of stored electrical energy into directed kinetic energy.

Previous work has shown ${ }^{4}$ that in an efficient pulsed inductive accelerator $\alpha$ should be between 1 and 3 . We choose 3 as a target value for our calculations based on the expected mass distribution in front of the coil. In addition, we set a target exhaust velocity of $25 \mathrm{~km} / \mathrm{s}$ and a thrust efficiency $\eta_{t}$ of $50 \%$. For an efficient pulsed electromagnetic accelerator, the value of $L^{*}$ should be less than unity. We felt that a value of 0.1 was realizable in the current configuration, and based our analysis on $L_{C}=700 \mathrm{nH}$ and $L_{0}=70 \mathrm{nH}$. Finally, measurements indicate that for our coil dimensions, the electromagnetic coupling distance $z_{0}$ is roughly 3.7 $\mathrm{cm}$. Using these numbers, we find that the unloaded circuit natural period is $5.1 \mu$ s and the capacitance required to given this period is $9.4 \mu \mathrm{F}$. Our capacitor bank is a modular design and each capacitor has a value of $2 \mu \mathrm{F}$, so we choose our target value $C=10 \mu \mathrm{F}$.

\section{B. Current Sheet Formation}

When the current pulse in the induction coil is initiated, it must transform the preionized plasma into a highly ionized, magnetically impermeable current sheet with essentially no delay. If this does not happen, the magnetic field produced by the induction coil will rapidly diffuse through the highly resistive plasma and radiate away, performing little or no work on the propellant. It was recently proposed ${ }^{3,5}$ that formation of a current sheet capable of supporting efficient acceleration will occur if the linear current density rise rate in the coil is roughly $10^{12} \mathrm{~A} /(\mathrm{m} \mathrm{s})$. If the coil is electrically connected to a single capacitor bank and is comprised of multiple spirals each making $n$ turns, then the initial current rise rate required from the bank is

$$
\left.\frac{d I}{d t}\right|_{t=0}=\left.\frac{\mathcal{L}}{n} \frac{d j}{d t}\right|_{t=0}
$$

where $d j / d t$ is the linear current density rise rate and $\mathcal{L}$ is equal to the distance from the inner to the outer radius of the coil (the length over which the linear current density is defined). Following this argument, the powertrain must produce an initial current rise rate equal to $45 \mathrm{kA} / \mu \mathrm{s}$. If we assume the acceleration coil is being driven by a powertrain approximated by an RLC circuit, then the initial current rise rate is $V_{0} / L_{0}$. Furthermore, if $L_{0}=70 \mathrm{nH}$, the initial capacitor charge voltage $V_{0}$ should be at least $3150 \mathrm{~V}$.

We summarize our target design parameters in Table 1.

Table 1. Summary of target FARAD thruster design parameters.

\begin{tabular}{ll|ll}
\hline$v_{z}$ & $25 \mathrm{~km} / \mathrm{s}$ & $L_{0}$ & $70 \mathrm{nH}$ \\
$\eta_{t}$ & $50 \%$ & $L_{C}$ & $700 \mathrm{nH}$ \\
Energy & $100 \mathrm{~J} /$ pulse & $z_{0}$ & $3.7 \mathrm{~cm}$ \\
$\alpha$ & 3 & $C$ & $10 \mu \mathrm{F}$ \\
Coil ID & $12 \mathrm{~cm}$ & $V_{0}$ & $\geq 3150 \mathrm{~V}$ \\
Coil OD & $30 \mathrm{~cm}$ & & \\
\hline
\end{tabular}

3 of 11 


\section{Thruster Design}

In the present FARAD thruster iteration, there are three major subsystems: propellant injection, preionzation, and acceleration. Each of these are discussed in turn below. Unless otherwise noted, these components. are being fabricated or integrated by Radiance Technologies.

\section{A. Propellant Injection}

The propellant injection system consists of two main components. These are a high speed gas valve from Applied Pulsed Power to pulse the gas into the system and a manifold designed to direct the radial flow outward across the surface of the thruster and distribute it as evenly as possible. The estimated distribution time for argon gas across the drive coil is $0.24 \mathrm{~ms}$.

The gas injection system uses a high speed valve (see Fig. 1A) with a radial orifice capable of generating gas pulses as short as $70 \mu \mathrm{s}$ in pulse width. This valve employs a low mass beryllium copper Belleville spring diaphragm that lifts off the seal at its outer diameter permitting gas to flow radially from the valve when open. The diaphragm is driven by a pancake solenoid, which is powered by solid-state switched $10 \mathrm{kA}, 3 \mathrm{kV}$ pulse generator. Continuous mode operation at 5 pulses per second or burst-pulse mode operation at 100 $\mathrm{Hz}$ can be achieved without forced cooling. The expected lifetime of the valve is $10^{8}$ pulses.
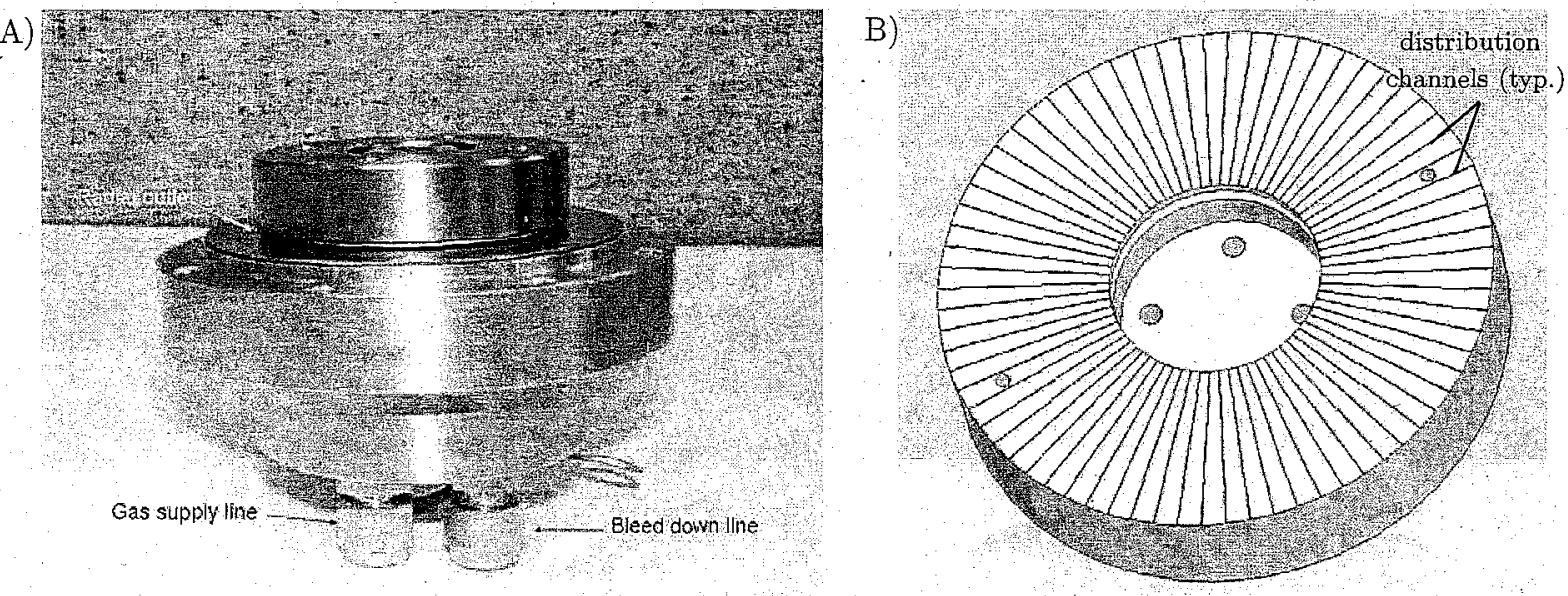

Figure 1. A) High speed valve. B) Rendering of the gas distribution manifold.

The manifold, seen in Fig. 1B, is fabricated from Macor and consists of 72 semicircular channels each $0.127 \mathrm{~mm}$ in radius. The manifold material was chosen to support machining of the small gas distribution channels. Macor has the added benefit of having a high temperature range and hardness, both of which should make it more resistant to degradation in the presence of the ionized propellant. The combined cross sectional area of the channels was chosen to set the mass flow rate to roughly $625 \mathrm{mg} / \mathrm{s}$ based upon distributing $150 \mu \mathrm{g}$ of argon gas over the drive coil in $0.24 \mathrm{~ms}$.

When the valve is opened, gas expands radially into the manifold channels. The channels are short enough relative to their diameter to ensure that wall friction has a minimal effect on the mass flow rate. The gas in the manifold does not begin to supersonically expand until it reaches the manifold exit where the Mach number is unity. Restricting the gas flow until it is at a larger radius (namely the ID of the acceleration coil) keeps the propellant closer to the drive coil over the entire radial range. Limiting the flow in the manifold to subsonic speeds also increases the distribution time in comparison to a completely supersonic expansion, resulting in more time between the initiation of the gas pulse and the other pulses in the system.

Once the gas distribution system has been "built, a fast ionization gauge will be used to measure the timevarying propellant distribution over the acceleration coil face. This measurement will also help in estimating and adjusting the mass injected during each pulse. 


\section{B. Preionization}

An antenna assembly is used to capacitively couple energy into the propellant for the purpose of creating the preionized plasma. The antenna is connected to a vector inversion generator (VIG), which supplies energy in the form of a short, pulsed-RF signal. ${ }^{7,8}$ Gas ionization experiments have shown that a significant portion of the propellant can be ionized for up to $20 \mu$ s using a VIG operating at as little as $30 \mathrm{~mJ}$ of input energy.

The elements of the VIG are shown schematically in Fig. 2A and B, and a VIG potted in castrol oil is shown in Fig. 2C. The lines shown in the schematics are metallic foils and the space between them is dielectric film. In this spiral configuration, the VIG consists of two parallel plate transmission lines wound on a mandrel and sharing a common conductor. During operation, the array is dc charged to approximately $2 \mathrm{kV}$ with energy stored in the capacitance of the spiral windings, $\mathrm{C}_{\mathrm{vig}}$. The erection sequence is initiated by closing the input switch, $\mathrm{S}_{1}$.
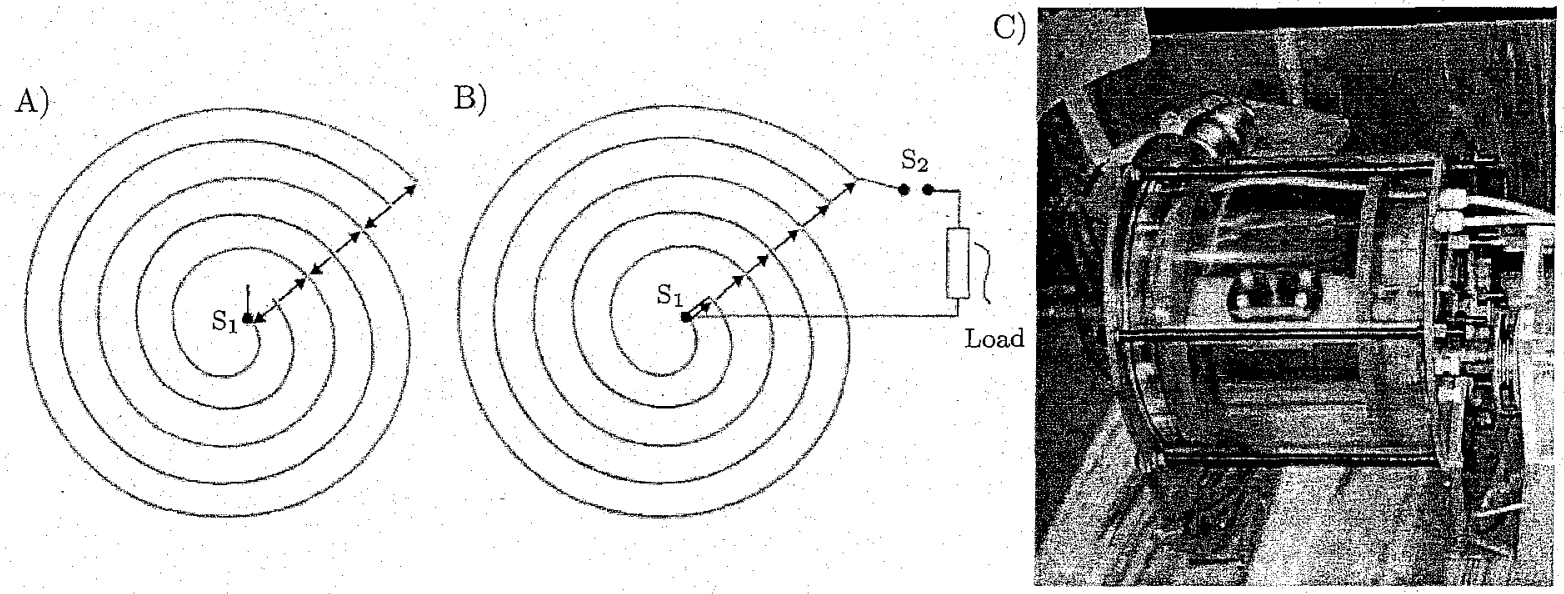

Figure 2. Schematic of a spiral line VIG A) DC charged and B) fully erected. Solid lines are metal foils and the intermediate spaces are insulators. Arrows represent the electric field vectors in each configuration. $C$ ) $A$ VIG potted in castrol insulating oil.

When switch $\mathrm{S}_{1}$ closes, an electromagnetic wave begins traveling up one of the strip lines. As a firstorder approximation, due to the inductance disparity between the two conductors, a wave does not propagate quickly in the second line. The net result is that the VIG is comprised of two LC circuits - one "fast" and one "slow". The electric field vectors plotted in Fig. 2a transition to the fully-erected configuration shown in Fig. $2 \mathrm{~b}$ after the "fast" wave travels to the "open end" and back to switch $\mathrm{S}_{1}$. If the transit time in the fast circuit is sufficiently short when compared to the slow circuit, the voltage across the array will be close to the ideal output of $2 n V_{0}$, where $V_{0}$ is the initial charge voltage and $n$ is the number of turns in the VIG. The net result is a transient high voltage between the lines at $S_{1}$ and the outermost turn on the VIG. The output voltage waveform is a ramp function with rise-time equal to the transit time up and back in the active line. Voltage outputs as great as $95 \%$ of ideal have been realized in certain geometries.

\section{Acceleration}

\section{Drive Coil Design}

The coil design was performed at NASA-MSFC and is a critical element in fixing the overall performance of the FARAD thruster. A geometry consisting of several interwoven spiral elements is chosen as this yields a current density over the coil face that is approximately uniform. This leads to a relatively uniform radial magnetic field between the acceleration coil and the induced current sheet. The radially uniform field results. in approximately equal axial acceleration profiles at any radial location in the current sheet, leading to full utilization of the propellant mass injected over the coil. To approach the performance levels assumed in Sect. II, the coil's inductance and acceleration stroke length must be close to those given in Table 1. The coil inductance is important when one considers that the ratio of the coil inductance to the stray (non-coil) inductance in the circuit is representative of the amount of energy that can be electromagnetically deposited into acceleration of the plasma. 
We surveyed several inductance approximation techniques in an attempt to find one that was appropriate for evaluating the inductance of a spiral acceleration coil like the one found in FARAD. These techniques included the Wheeler and modified Wheeler inductance formulas, ${ }^{9}$ an approximation based on an azimuthal current sheet model, the use of conformal modules, ${ }^{10}$ an approximation based on a flat spiral coil, ${ }^{11}$ and an expression based on a curve fit of experimental data. The approximation techniques were able to predict the inductance of a single coil with relative precision. Unfortunately, they were not capable of modeling the inductance of a multiple-wire spiral coil possessing the dimensions of the FARAD thruster because of the addition of mutual inductance to the problem.

Because of low confidence in the inductance approximation techniques for the FARAD coil, we performed a series of experimental inductance measurements using the coil shown in Fig. 3. The coil possessed multiple two turn spirals with the first turn of each coil traversing from the outer to inner diameter and the second turn returning to the beginning of the first turn. The two turns were spatially located on different planes to permit the wires to cross without difficulty. In this setup, we could test any number of coils from a single two-turn spiral to every spiral in parallel.

We found that for two-turn spirals possessing an OD of $30 \mathrm{~cm}$ and an ID of $12 \mathrm{~cm}$, the agreement between the experimentally measured

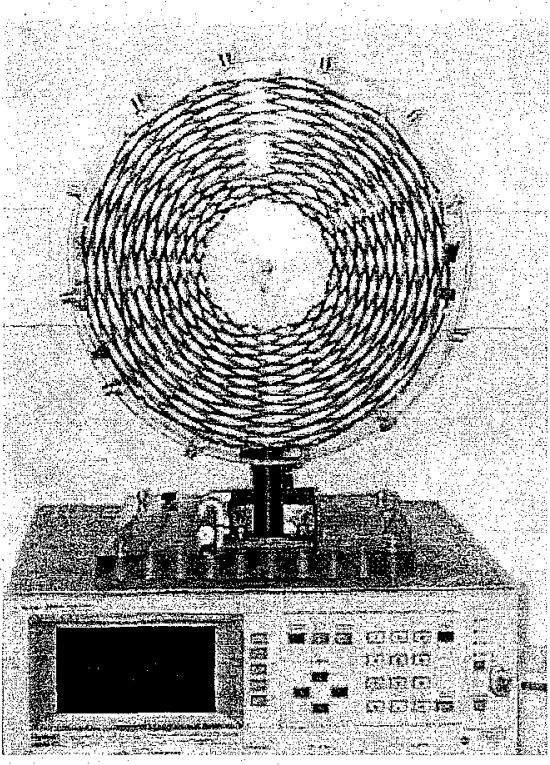

Figure 3. FARAD test coil comprised of 12 two-turn coils and having an $O D$ of $30 \mathrm{CM}$ and an ID of $12 \mathrm{~cm}$. coil inductance (that is, the inductance measured when the plasma is at $z=\infty$ ) and that predicted using a non-coupled theoretical treatment (see Fig. 4A) is generally poor. This comparison is representative of the poor agreement between our measurements and all the theoretical models.

From Figs. 4A and B, we see that the measured inductance is roughly constant for 3 or more spirals. Physically, this result implies that 3 two-turn spirals in our configuration produces a magnetic field topology that reasonably approximates one produced by a purely azimuthal, uniform current distribution over the coil face. We elected to build a coil comprised of 6 two-turn spirals for the final design to remove nonuniformities in the near-field of the coil. The measured coil inductance in this configuration was $719 \mathrm{nH}$.

A)

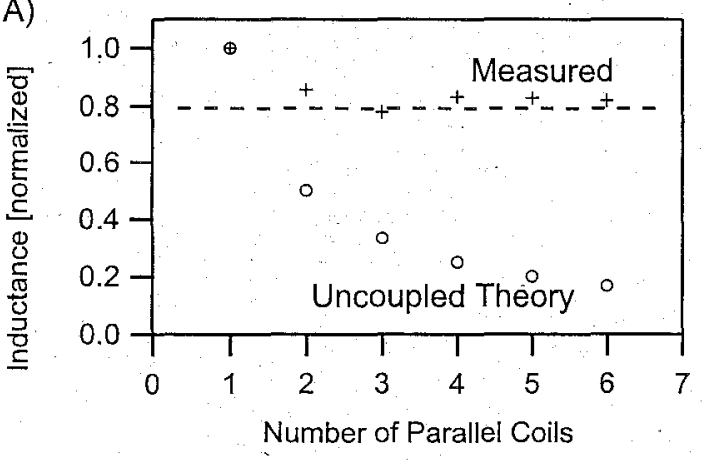

B)

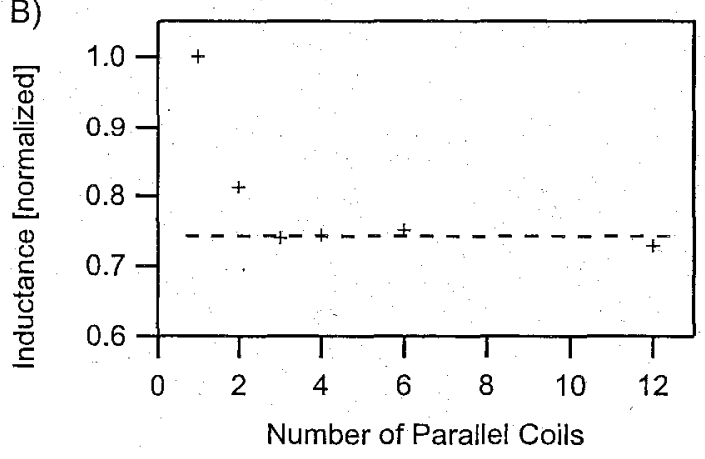

Figure 4. A) Comparison between the measured inductance (normalized) of a set of parallel, spiral coils and the predictions of a theoretical treatment assuming that the coils are uncoupled. B) Measured inductance (normalized) of a spiral coil as a function of the number of parallel coils used. Dashed lines inserted to demonstrate constancy of $L_{\text {coil }}$ for a greater number of coils.

In the actual thruster, the inductance the coil presents to the rest of the circuit varies as the plasma moves away from the coil. We measured the coil inductance as a function of plasma position by placing a conducting plate at different axial positions relative to the coil to approximate the effect of the plasma. Inductance was measured using an Agilent $4285 \mathrm{~A}$ precision LCR meter, which is capable of measuring inductance at frequencies from $75 \mathrm{kHz}$ to $30 \mathrm{MHz}$. Our data was collected primarily at $100 \mathrm{kHz}$ as this is 
close to the expected frequency of the current pulse in the FARAD. We found that the inductance presented to the circuit as a function of plasma axial position followed the well-known empirical scaling ${ }^{12}$

$$
L(z)=L_{0}+L_{C}\left[1-\exp \left(-z / z_{0}\right)\right]
$$

where $L$ is the total inductance measured in the circuit, $L_{0}$ is the stray inductance in the circuit, and $z_{0}$ is the electromagnetic decoupling length scale. In our final design, this length scale was $3.7 \mathrm{~cm}$, and in general our studies found that $z_{0}$ was always between 10 and $15 \%$ of the coil OD. A rendering of the final coil design is shown in Fig. 5.

In addition to our inductance measurements, we modeled the coil using the 2-D axisymmetric magnetostatic solver package in QuickField. We modeled the coil by taking a cross-sectional cut across the coil face and placing a circular conductor in every position in the $r-z$ plane where our cut intersected the coil. This is not strictly correct since the coil is not axisymmetric. However, our approximation should be valid so long as the magnetic field produced by the coil is close to that produced by a purely azimuthal, uniform current distribution at the coil face.

We find that for a two-turn, 6 coil geometry with the cut taken at a location where the coil windings cross each other, the computed inductance from our model is $882 \mathrm{nH}$. It is not unexpected that this number is greater than the one measured experimentally. The coil can be completely isolated in the model and its inductance calculated, but in the measurement the coil inductance is taken as the difference between the inductance when the metal plate is at

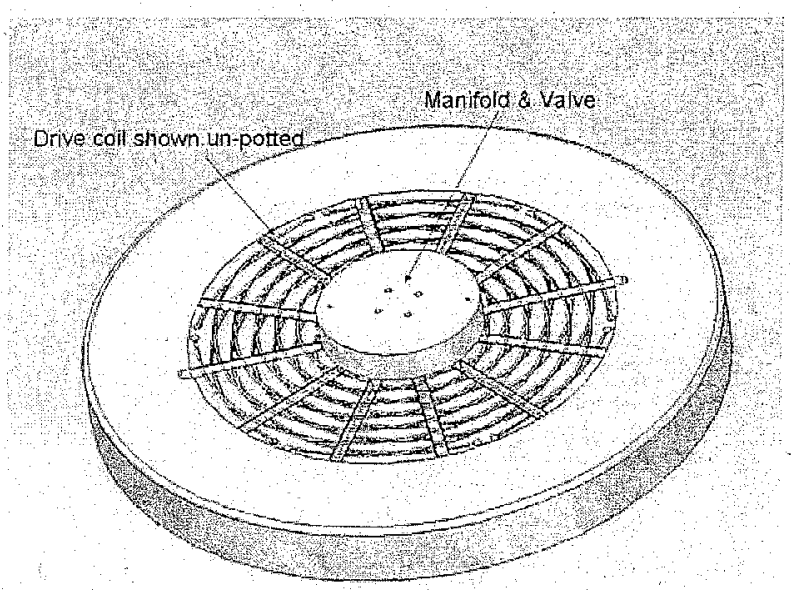
$z=\infty$ and when it is as close to the coil as physically possible. A small amount of the coil inductance, representing of the difference between the metal plate being as close as possible and the plate actually being colocated with the coil, is inaccessible for performing electromagnetic acceleration on the plasma. In the experimental results, this inaccessible coil inductance is accounted for as additional stray inductance. There are also physical irregularities in the experimental setup where the coils cross each other and where they connect to coaxial feeds at the OD of the coil.' A more refined assembly technique in the final FARAD acceleration coil should help to minimize the stray inductance attributable to these irregularities.

\section{Bernardes and Merryman Power Train}

The Bernades and Merryman (B\&M) circuit ${ }^{13}$ is a pulsed power train designed to allow for energy recapture. The basic B\&M circuit shown schematically in Fig. $6 \mathrm{~A}$ has two capacitor banks of equal capacitance. When the switch is closed, charge will oscillate between the two capacitor banks through the drive coil, represented in the schematic as $\mathrm{N} 1$. At the end of the transient the voltage and energy on capacitor banks $\mathrm{C}_{1}$ and $\mathrm{C}_{2}$ will be equal. In this scheme, capacitor bank $\mathrm{C}_{1}$ can be brought back to full charge for the next pulse. Meanwhile, bank $\mathrm{C}_{2}$ must be discharged before the next pulse is initiated. The excess energy on bank $\mathrm{C}_{2}$ could possibly to be used to power other spacecraft loads. This setup allows for recovery of at least half the energy remaining after the pulse transient. The target capacitance of $10 \mu \mathrm{F}$ required for acceleration optimization (see Sect. II) is given by the series configuration of the $20 \mu \mathrm{F}$ comprising both banks $\mathrm{C}_{1}$ and $\mathrm{C}_{2}$. However, the energy stored for each pulse is equal to $1 / 2\left(\mathrm{C}_{1}\right) V_{0}^{2}$. A target energy of $100 \mathrm{~J} / \mathrm{pulse}$ is obtained for a charge voltage of $3160 \mathrm{~V}$, which is slightly greater than the voltage required for current sheet formation.

Example current and voltage waveforms for the $B \& M$ circuit are presented in Fig. $6 B$. These measurements demonstrate an additional advantage of the $B \& M$ circuit, namely that it precludes voltage reversal on either capacitor bank. This reduces the stress on the capacitors and increases their operational lifetimes. 

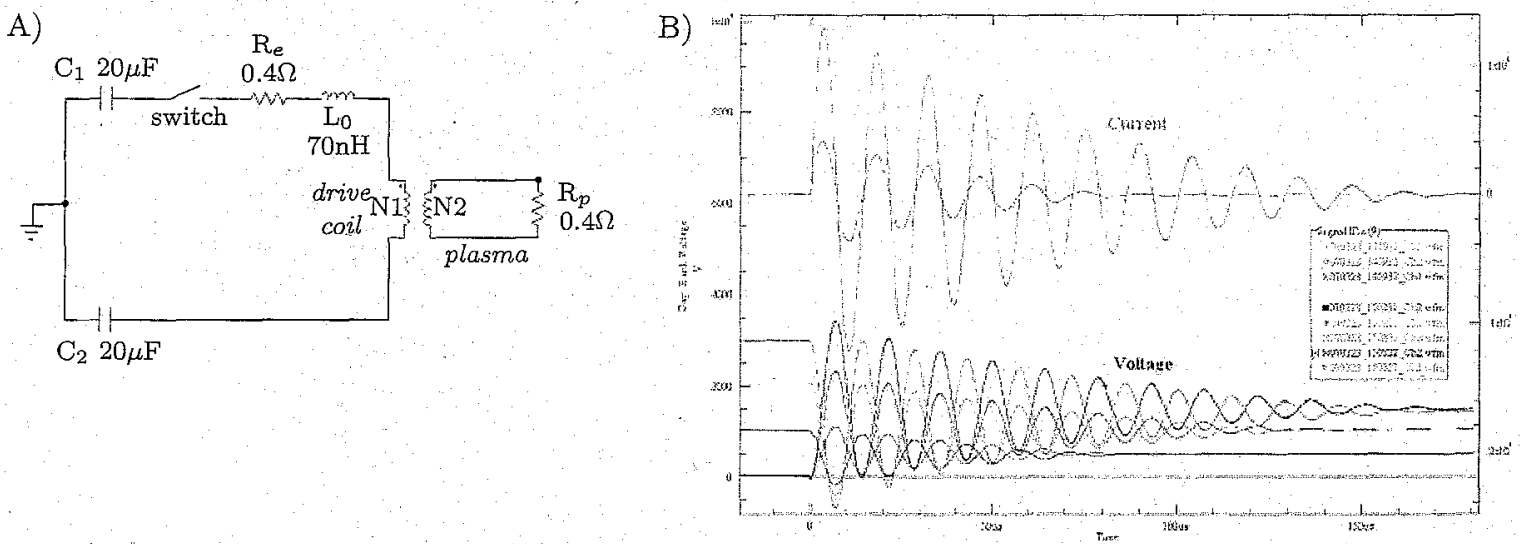

Figure 6. A) B\&M circuit schematic for the FARAD thruster. B) B\&M voltage and current waveforms into a $540 \mathrm{nH}$ inductive load for 1,2 , and $3 \mathrm{kV}$ initial capacitor charge.

To improve thruster lifetime relative to other pulsed inductive concepts that have been switched using spark gaps, ${ }^{1}$ a solid-state switch manufactured by Applied Pulsed Power was selected for integration into the FARAD B\&M circuit. To date, it has proved capable of switching $3 \mathrm{kV}$ applied to bank $\mathrm{C}_{1}$ in the $\mathrm{B} \& \mathrm{M}$ circuit shown in Fig. 6 and discharged through a $540 \mathrm{nH}$ inductive load with a peak current of $13 \mathrm{kA}$ (see Fig. 6B). In the final FARAD thruster, the switch must be capable of switching a $3.2 \mathrm{kV}$ capacitor bank charge with a peak current over $30 \mathrm{kA}$. It must also possess low inductance so as to not place the thruster above the design target of $70 \mathrm{nH}$ stray inductance. The capabilities of the switch have not yet been evaluated with respect to the FARAD thruster requirements.

To further improve upon the B\&M circuit's energy recovery potential, a latching design is also being studied. A circuit schematic of this concept in shown in Fig. 8A, where latching diodes have been

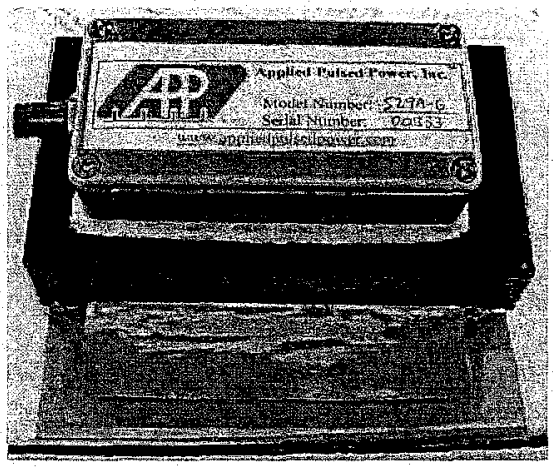

Figure 7. Applied Pulsed Power solidstate switch. added in parallel to two solid-state switches; one for each capacitor bank. It is well-known that plasma acceleration in a pulsed inductive plasma accelerator is most effectively accomplished during the first half-cycle, where coil current and $d I / d t$ levels are greatest. Beyond that, a significant amount of energy is wasted in resistive heating of the external circuit and production of a magnetic field that radiates into space. The diodes in Fig. $8 \mathrm{~A}$ serve to reduce the amount of energy wasted in the discharge by allowing current to flow in the circuit for only the first half-cycle of the discharge. During the discharge, the initially uncharged capacitor bank is partially charged by the current flowing through the coil from $\mathrm{C}_{1}$ to $\mathrm{C}_{2}$. This reduces the amount of charge we must apply to bank $\mathrm{C}_{2}$ before the next pulse, effectively recapturing energy that would otherwise have been lost in the system.

A diode-latched design was bench tested with an inductive load equivalent to approximately $570 \mathrm{nH}$. The voltage and current waveforms from this test are shown in Fig. 8B. For a non-latching B\&M circuit, the remaining energy would be equally split between the capacitor banks. In the latching B\&M test circuit, a larger percentage of the remaining energy in the circuit was recaptured by the second, initially uncharged capacitor bank. Testing is currently in progress to determine if this alternative design can be used at the charge voltage and peak current levels required in the FARAD thruster.

\section{Pulse Compression Power Train}

A pulse compression system developed by the West Virginia High Technology Consortium Foundation provides an attractive alternative means of powering the FARAD thruster. In this power train, initially slow, low-current pulses are compressed to yield short, high-current pulses. Solid-state switching is employed prior to pulse compression to keep current levels relatively low at the primary switching element. Typical 
A) $\mathrm{C}_{1} 20 \mu \mathrm{F}$.

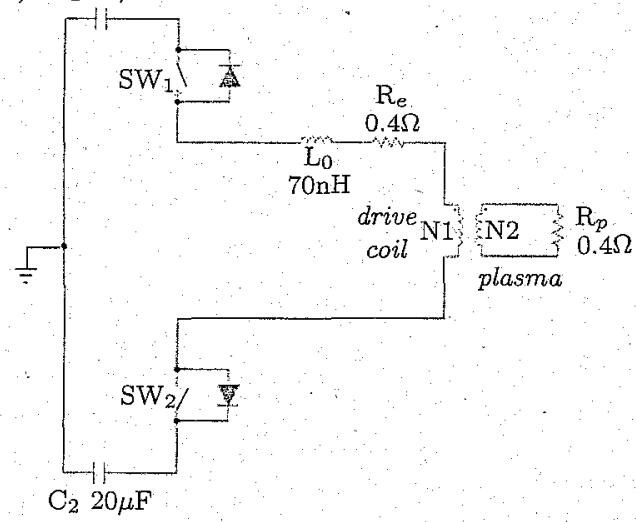

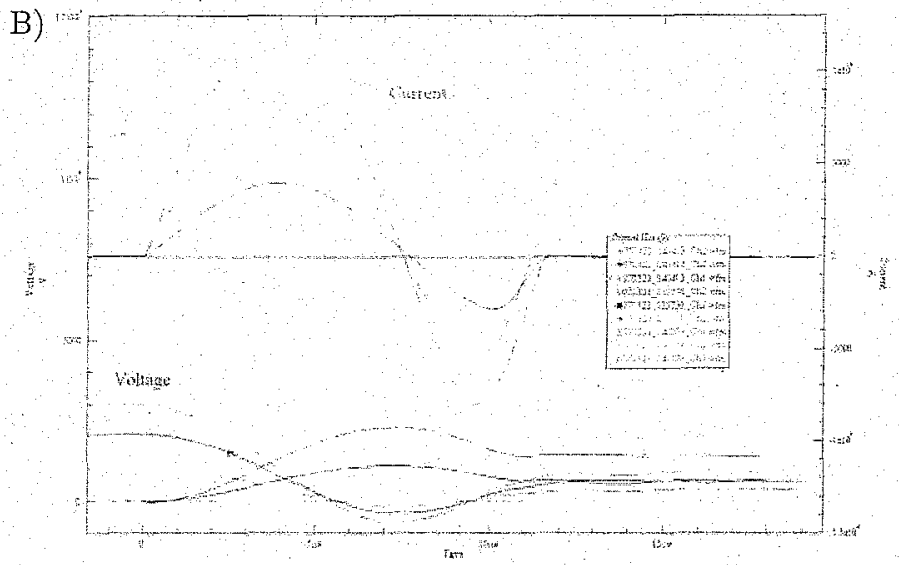

Figure 8. A) Diode-latched B\&M circuit schematic for the FARAD thruster. B) Diode-latched B\&M voltage and current waveforms for 1,2 , and $3 \mathrm{kV}$ initial capacitor charge.

pre-compressed currents are low enough to allow the use of fast IGBT switches. The high pulse-repetition rates possible with IGBT switches lead to attractive values for thruster specific mass $(\mathrm{kg} / \mathrm{kw})$.

A PSPICE model for the pulse compression circuit considered for this project is shown in Fig. 9A. Current from the primary energy-storage capacitor $\mathrm{C} 1$ is compressed a factor of five prior to application at the output capacitor $\mathrm{C} 3$. The resulting fast charge of capacitor $\mathrm{C} 3$ leads to low volt-seconds at the output magnetic switch L3. Low volt-seconds keep the core size for the magnetic switch reasonably small, as demonstrated using the integral form of Faraday's law,

$$
A_{c}=\frac{1}{2 n B_{s}} \int V d t
$$

where $A_{c}$ is the area of the magnetic switch core, $n$ is the number of turns made by the conductor wrapped around the core, $V$ is the voltage applied to the conductor, and $B_{s}$ is the saturation magnetic field in the core. The magnetic switch can handle current amplitudes and rates-of-change of current well above levels tolerated by traditional solid-state switches. The drive coil for the thruster is represented in the model by the inductor $\mathrm{L}$ Coil and the resistor R3. The circuit inductance can be approximately transformed from the spatially-dependent form given in Eq. (4) to a temporally-varying form by using the substitutions $z=v_{z} t$ and $z_{0}=v_{z} t_{0}$. This yields

$$
L(t)=L_{0}+L_{C}\left[1-\exp \left(-t / t_{0}\right)\right]
$$

where $L_{0}$ is the stray drive coil inductance at $t=0, L_{0}+L_{C}$ is the total drive coil inductance in vacuum, and $t_{0}$ is the characteristic time for plasma acceleration in the field of the drive coil. The time-dependent drive-
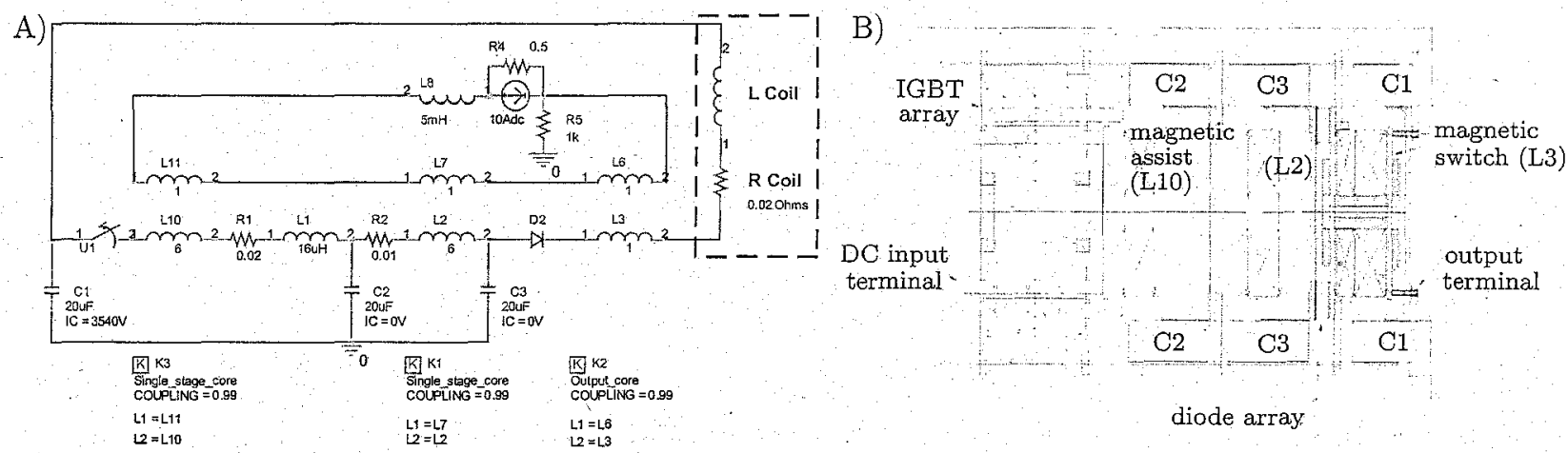

Figure 9. A) PSPICE schematic model and B) hardware implementation of the pulse compression ring circuit. 
coil inductance is included in the simulation using the analog behavior models (not shown in the schematic) within PSPICE.

The circuit depicted in Fig. 9A uses a novel energy-recovery scheme in which current passing through the drive coil is circulated back into the initial energy storage capacitor $\mathrm{C} 1$ through a resonant transfer from $\mathrm{C} 3$ to C1. Energy is held in C1 by means of steering diode D2. In effect, the circuit forms a pulse compression ring topology in which energy flows in only one direction around the circuit. This topology eliminates voltage reversal on the capacitive elements, resulting in a substantial increase in capacitor lifetime. Furthermore, recovered energy does not have to make a second transit of the circuit, as in other pulse compressors. ${ }^{14}$ This feature leads to improved energy efficiency.

Guided by PSPICE simulations, we are presently assembling the pulse compression ring shown schematically in Fig. 9B. The solid-state switching element in the circuit is formed from a circular array of eight IGBTs. The magnetic assist L10 is composed of eight independent windings on a single magnetic core. Each winding is connected to an individual IGBT. This construction assures uniform turn-on of all eight IGBTs and uniform current sharing between IGBTs. Steering diode D2 is formed from an array of six fast-response power diodes. The output magnetic switch L3 acts as a magnetic assist for this diode array to assure simultaneous turn-on of the array, low turn-on losses, and uniform current-sharing between individual diodes.

\section{Design Summary}

We have presented the design of a complete FARAD thruster that is optimized based on previously derived performance scaling parameters and experimentally determined design rules. The thruster is designed to operate at a discharge energy of $100 \mathrm{~J} /$ pulse, and we have performed our design analysis using a target exhaust velocity and thrust efficiency of $25 \mathrm{~km} / \mathrm{s}$ and $50 \%$, respectively. Propellant is supplied to the thruster using a multi-channel gas distribution manifold that is fed by a high-speed, pulsed gas valve. Preionization is accomplished using a pulsed-RF signal supplied by a VIG. The coil has an OD of $30 \mathrm{~cm}$ and an ID of 12 $\mathrm{cm}$ and is comprised of 4 two-turn spirals, with each conductor spiraling in on the first turn and back out on the second turn. Measurements and finite-element modeling have shown that a coil of this design will meet the target inductance of at least $700 \mathrm{nH}$. For the coil design, we have set our target stray (non-coil) inductance in the circuit equal to $70 \mathrm{nH}$ to support both efficient acceleration and transformation of the preionized plasma into a highly-ionized, magnetically impermeable current sheet at $t=0$. The current is driven through the acceleration coil using either a Bernardes and Merryman pulsed power train or a pulse compression power train. Both power trains are switched using solid-state components, precluding the need for spark gap switches that are prone to erosion over time.

\section{Acknowledgments}

This work was performed under NASA contract NNM06AA17G, managed by Dr. Michael LaPointe, supporting the West Virginia High Technology Consortium Foundation's Pulsed Plasma Accelerator (PPA06) program.

\section{References}

${ }^{1}$ C.L. Dailey and R.H. Lovberg, The PIT MkV Pulsed Inductive Thruster, Tech. Rep. NASA CR-191155, TRW Systems Group, July 1993.

2E.Y. Choueiri and K.A. Polzin, "Faraday Acceleration with Radio-frequency Assisted Discharge," J. Propuls. Power, 22(3):611, May-June 2006.

${ }^{3}$ K.A. Polzin, Faraday Accelerator with Radio-frequency Assisted Discharge, Ph.D. Dissertation, 3147-T, Princeton Univ., Princeton, NJ, 2006.

${ }^{4}$ K.A. Polzin and E.Y. Choueiri, "Performance Optimization Criteria for Pulsed Inductive Plasma Acceleration," IEEE Trans. Plasma Sci., 34(3):945, June 2006.

${ }^{5}$ K.A. Polzin and E.Y. Choueiri, ."Design Rules for High-Performance FARAD Thrusters," 29th Int'l Electric Propuls. Conf., Princeton, NJ, Oct.-Nov, 2005. IEPC Paper 2005-207.

${ }^{6}$ R.H. Lovberg, B.R. Hayworth, and T. Gooding, The use of a coaxial plasma gun for plasma propulsion, Tech. Rep. AE62-0678, G.D. Convair, May 1962.

${ }^{7}$ A. Ramrus and M.F. Rose, "High-Voltage Spiral Generators," Proc. 1st IEEE Int'l Pulsed Power. Conf., T.R. Burkes and M. Kristiansen, ed, Lubbock, TX, 1976. Paper IIIC-9. 
${ }^{8}$ J.W. Rice, R.J. Gripshover, M.F. Rose, and R.C. Van Wagoner, "Spiral line oscillator," U.S. Patent 4,217,468, 1980.

${ }^{9}$ S.S. Mohan, M. del Mar Hershenson, S.P. Boyd, and T.H. Lee, "Simple Accurate Expressions for Planar Spiral Inductances," IEEE J. Solid-State Circuits, 34(10):1419, Oct: 1999.

${ }^{10} \mathrm{~J}$. Zhao and J. Mao, "Inductance Extraction for Planar Spiral Inductor by Domain Decomposition Method for Conformal Modules," IEEE MTT-S Digest, pp. 1203-1205, 2003.

${ }^{11}$ F.W. Grover, Inductance Calculations: Working Formulas and Tables, Dover Publications, Inc., New York, 1946.

${ }^{12}$ R.H. Lovberg and C.L. Dailey, "Large inductive thruster performance measurement," AIAA J., 20(7):971, July 1982.

${ }^{13} \mathrm{~J}$. Bernardes and S. Merryman, "Parameter Analysis of a Single Stage Induction Mass Driver," Proc. 5th IEEE Int'l Pulsed Power Conf., M.F. Rose and P.J. Turchi, ed,, pp. 552-555, Arlington, VA, 1985. Paper PI-27.

${ }^{14}$ D.L. Birx, P.P. Das, I.V. Fomenkov, W.N. Partlo, and T.A. Watson, "Pulse power generating cireuit with energy recovery," U.S. Patent 5,729,562, 1998 . 